\title{
Theranostics
}

Editorial

2012; 2(9):815-816. doi: 10.7150/thno.5128

\section{Porphyrins and Phthalocyanines for Theranostics}

\section{Jonathan F. Lovell ${ }^{1,2 \varpi}$, Pui-Chi Lo ${ }^{3}$}

1. Department of Chemical and Biological Engineering, University at Buffalo, State University of New York, Buffalo, NY 14260, USA;

2. Department of Biomedical Engineering, University at Buffalo, State University of New York, Buffalo, NY 14260, USA;

3. Department of Chemistry, The Chinese University of Hong Kong, Shatin, N.T., Hong Kong

$\triangle$ Corresponding author: jflovell@buffalo.edu

() Ivyspring International Publisher. This is an open-access article distributed under the terms of the Creative Commons License (http://creativecommons.org/ licenses/by-nc-nd/3.0/). Reproduction is permitted for personal, noncommercial use, provided that the article is in whole, unmodified, and properly cited.

Published: 2012.10 .04

The field of theranostics is growing rapidly as therapeutic regimes are increasingly being integrated and guided by diagnostic practices such as medical imaging. There is some room for discussion about the general definition of a theranostic agent, if there exists such a definition. Is it a diagnostic imaging agent that is used in the context to guide surgical procedures? Or rather, is it a therapeutic agent whose administration and dosing is guided by diagnostic metrics? Probably, theranostics encompasses both of these concepts and many additional ones, which are regularly discussed within this journal. However, it may be presumed that single agents which have the capacity for both imaging and therapy are particularly suited for theranostic applications. Porphyrins and phthalocyanines, which share an aromatic and planar tetrapyrrole backbone, are one class of such molecules which are ideally suited for intrinsic multifunctional imaging and therapeutic applications. In this special Theranostics issue, which is dedicated to porphyrins and phthalocyanines, a partial representation of the multifunctional theranostic capabilities of porphyrins and phthalocyanines is presented.

The incredibly rich history of porphyrins and phthalocyanines as theranostics agents is presented in a review paper within this issue [1]. The endogenous bright red porphyrins in blood could be considered as the original old theranostic agent. Heme has maintained its use in new imaging techniques as the central molecule that enables functional magnetic resonance imaging. Porphyrins have been pioneering agents for modern theranostics. Heme derivatives have been used for cancer imaging as early as the 1920s, phthalocyanines were used for positron emission studies since the 1950s and porphyrins have been used as MR contrast agents since the 1980s. Of course, porphyrins have made a clinical mark on modern theranostics in the form of photodynamic therapy (PDT). Most porphyrins generate singlet oxygen upon irradiation, so they can be used for therapeutic applications involving a photosensitizer, light and oxygen. A definitive, comprehensive summary of the theranostic roles of porphyrins with respect to photodynamic therapy is presented as a review in this issue [2].

The intertwined fluorescent and photodynamic properties of porphyrins permit novel optical theranostic approaches. By using two-photon fluorescence lifetime imaging, Yeh et al. demonstrate that subcellular localization can be determined based on differences in porphyrin fluorescence lifetime properties in the cell membrane and the cytosol [3]. By developing such novel cutting-edge optical techniques, it is hoped that such methodologies and instrumentation may eventually enter clinical practice for improving routine PDT. PDT is a multi-factor treatment, with clinicians being able to control laser power, laser treatment time, drug dose, and number of laser treatments. Thus, developing feedback systems to guide therapy is critical. Glidden et al., use image-based quantification in a three dimensional tumor model to optimize PDT parameters [4]. By understanding the interplay between drug localization, 
drug bleaching during treatment, and cellular response, PDT treatment variables can be better understood and optimized. In another study illustrating the utility of the multimodal parameters of porphyrins, Mitra et al. use the dual fluorescent/photodynamic properties of a photosensitizer to examine drug uptake, along with imaging of perfusion and immune response, to better more thoroughly determine the outcome of tumors treated with PDT [5].

Although porphyrins and phthalocyanines share a tetrapyrollic backbone, there are literally unlimited variations on substituent components of porphyrins. In addition, by inserting a chelated metal within the porphyrin, even more possibilities exist. In this issue, Ongarora et al. use new phthalocyanine chemistry to create a series PEGylated cationic molecules with promising in vitro behavior against cancer cells [6]. Based on the fluorescent and photodynamic properties of the chemical derivatives, both uptake and therapy could be readily characterized. Rather than synthesizing new types of phthalocyanine molecules, Lim et al developed a novel nanocarrier formulation to effectively deliver phthalocyanines to tumors in vivo [7]. Following accumulation in the tumor, photothermal therapy (rather than photodynamic) was used to achieve therapeutic effect.

Yumita et al. present a novel report of using sound, rather than light, to achieve destruction of cancer cells using a novel porphyrin derivative [8]. The mechanism, which was demonstrated to be related to the generation of singlet oxygen, has not been extensively explored by many research groups but offers compelling upside due to the superior travel of sound waves through body tissue compared to light waves. Finally, Benachour et al. present a compelling example of a multimodal, targeted nanoparticle formulation that was used for both MR imaging and chlorin-mediated PDT for brain cancer applications [9].

These review and research articles are illustrative of some of the exciting theranostic capabilities of porphyrins and phthalocyanines. Without doubt, future translational research will continue to use these multifunctional molecules for new theranostic applications.

\section{References}

1. Zhang $Y$, Lovell JF. Porphyrins as Theranostic Agents from Prehistoric to Modern Times. Theranostics 2012; 2(9):905-915.

2. Josefsen LB, Boyle RW. Unique Diagnostic and Therapeutic Roles of Porphyrins and Phthalocyanines in Photodynamic Therapy, Imaging and Theranostics. Theranostics 2012; 2(9):916-966

3. Yeh SCA, Diamond KR, Patterson MS, Nie Z, Hayward JE, Fang Q. Monitoring Photosensitizer Uptake Using Two Photon Fluorescence Lifetime Imaging Microscopy. Theranostics 2012; 2(9):817-826.

4. Glidden MD, Celli JP, Massodi I, Rizvi I, Pogue BW, Hasan T. Image-Based Quantification of Benzoporphyrin Derivative Uptake, Local- ization, and Photobleaching in 3D Tumor Models, for Optimization of PDT Parameters. Theranostics 2012; 2(9):827-839.

5. Mitra S, Mironov O, Foster TH. Confocal Fluorescence Imaging Enables Noninvasive Quantitative Assessment of Host Cell Populations In Vivo Following Photodynamic Therapy. Theranostics 2012; 2(9):840-849.

6. Ongarora BG, Hu X, Verberne-Sutton SD, Garno JC, Vicente MGH. Syntheses and Photodynamic Activity of Pegylated Cationic Zn(II)-Phthalocyanines in HEp2 Cells. Theranostics 2012; 2(9):850-870.

7. Lim CK, Shin J, Lee YD, Kim J, Oh KS, Yuk SH, Jeong SY, Kwon IC, Kim S. Phthalocyanine-Aggregated Polymeric Nanoparticles as Tumor-Homing Near-Infrared Absorbers for Photothermal Therapy of Cancer. Theranostics 2012; 2(9):871-879.

8. Yumita N, Iwase $Y$, Nishi K, Komatsu H, Takeda K, Onodera K, Fukai T, Ikeda T, Umemura Si, Okudaira K, Momose Y. Involvement of Reactive Oxygen Species in Sonodynamically Induced Apoptosis Using a Novel Porphyrin Derivative. Theranostics 2012; 2(9):880-888.

9. Benachour $\mathrm{H}$, Sève $A$, Bastogne $T$, Frochot $C$, Vanderesse R, Jasniewski J, Miladi I, Billotey C, Tillement O, Lux F, Barberi-Heyob M. Multifunctional Peptide-Conjugated Hybrid Silica Nanoparticles for Photodynamic Therapy and MRI. Theranostics 2012; 2(9):889-904. 